\title{
Krieg und Frieden an der Unteren Donau
}

\author{
Siedlungsgeschichtliche und demographische Bemerkungen über die \\ Kaza Ziştova - Svištov 1460-1878 anhand osmanischer administrativer \\ Quellen $^{1}$
}

MACHIEL KIEL

In den letzten 40 Jahren ist über die osmanische Siedlungspolitik auf dem Balkan, über die demographische Geschichte und ethnisch-religiöse Veränderungen manches gesagt und geschrieben worden. In den meisten Fällen ist dies mit viel zu wenigen Quellen geschehen und, schlimmer, aus einer sehr verzerrenden nationalistischen Perspektive, die uns mehr über die Denkweise des Verfassers und seiner politischen Präferenzen erzählt als über die historischen Ereignisse. Die Geschichte der demographischen Entwicklungen und der Siedlungen auf dem Balkan kann und darf nicht geschrieben werden, ohne die reichhaltigen osmanischen administrativen Quellen in ihrer Gesamtheit heranzuziehen. Eine solche Darstellung darf auch nicht von osmanistischen Philologen verfasst werden, die mit Scheuklappen die Mechanismen der historischen Demographie betrachten und die reichhaltige Literatur $\mathrm{zu}$ diesem Thema überhaupt nicht kennen, nicht verwenden oder nicht verwenden wollen. Auch können solche Forschungen nicht von jemandem gemacht werden, der das Gelände nicht selbst aus eigener Anschauung kennt.

Um an einem Beispiel zu illustrieren, was machbar ist, wenn man alle Möglichkeiten kombiniert, wollen wir hier eine Übersicht über die Entwicklung eines Gebiets in Donau-Bulgarien zu geben versuchen, wo die Quellenlage sehr günstig ist. Seit dem Mittelalter hat es in Donau-Bulgarien drei tiefe, durch Kriege und Zerstörungen verursachte Einschnitte gegeben. Sie sind aus der er-

1 Die dieser Arbeit zu Grunde liegenden Forschungen im Osmanischen Archiv in Istanbul, im Katasteramt in Ankara und in der Orientalischen Sammlung der Sofioter Nationalbibliothek sowie die eingehende Bereisung des Gebiets wurden im Rahmen des von der DFG unterstützten Münchner Ortnamenprojekts durchgeführt. Ein früheres Teilprojekt im gleichen Gebiet wurde durch Unterstützung der Z.W.O.-N.W.O, Den Haag, Niederlande,ermöglicht. Der türkischen und bulgarischen Archivverwaltung gebührt Dank für ihre oft unbürokratische Hilfe. The rich article of Mariana Drumeva, „Demografsko-ikonomičesiyat oblik na Svištovo do načalato Bălgarskoto Văzraždane“, in: Dialog, 4, 2010, p. 45-78, readed me to late to be incorporated in the present article. It deals with the town of Svištov, not with the villages of its District. 
zählenden Literatur bekannt. Wie sich das auf die demographische Entwicklung ausgewirkt hat, zeigen lediglich die osmanischen administrativen Quellen.

Unser Beispiel, die historische Kaza (Gerichtssprengel) Ziştova, umfasst ein Gebiet von etwa $50 \times 35$ km, das sich längs der Donau erstreckt und vom Fluss leicht zum Innenland hin ansteigt. Es besteht großenteils aus sehr fruchtbarem Flachland, durchzogen von kleinen natürlichen Erhebungen. Die Donaustadt Svištov war von alters her das einzige städtische Zentrum des Gebiets. Die Grenzen der Kaza sind seit dem Mittelalter ziemlich konstant geblieben. Nur eine kleine Gruppe Dörfer am Südrand, Habibli, Batak, Kara İsa gehörten bis zu den Reformen der Tanzimat zur Kaza Tirnova, und die Dörfer Bălgarene, Lădžene und Kozar Belene in der äußersten Südwestecke zur Kaza Niğbolu/Nikopol.

Schauen wir uns die Karten des 19. Jahrhunderts an, also aus der Zeit, bevor die massiven Umbenennungs- und Bulgarisierungskampagnen des 20. Jahrhunderts fast die gesamte historisch gewachsene Toponymie auslöschte, so ergibt sich ein kompliziertes Gemisch aus bulgarischen und türkischen Dorfnamen. ${ }^{2}$ Will man, ausgehend von den Dorfnamen, die ethnisch-religiöse Zugehörigkeit der Bewohner feststellen und zieht dazu die Angaben im Salname (Almanach) von 1873 zu Rate, das die Zahl der Haushalte nach Religionszugehörigkeit aufschlüsselt, so stellt sich heraus, dass diese Methode nur beschränkt funktioniert. So gab es 1873 die Dörfer Bunarlı, Çatma, Hacı Musa, Sarıyar und Yayc1 mit schönen alten türkischen Namen, aber mit einer mehrheitlich christlichen Bevölkerung. Kara İsa war fast ganz christlich. Dagegen gab es Dörfer mit gut bulgarischen Namen, aber mit ausschließlich muslimischen Einwohnern, wie Červena und Virzil/Vărzulica.

Beim Vergleich der Karten lässt sich auch feststellen, dass sich hinter bestimmten türkischen Ortsnamen ältere, bulgarische, verbergen. So finden wir für Bunar, auch Beş Bunar genannt, die ältere bulgarische Form Pet Kladenci (Fünfbrunnendorf) und bei Tekfur die ältere Form Tekfur Kladenec. In der ältesten Quelle aber heißt der Ort auf gut Bulgarisch Carev Kladenec (Kaiserbrunnen). Das im 19. Jahrhundert entstandene Dorf Biberli heißt auf Bulgarisch Piperkovo. Da sowohl Pet Kladenci als auch Piperkovo ganz oder mehrheitlich christlich waren, lässt sich behaupten, dass beides ursprünglich bulgarische Dörfer gewesen sind.

Um tiefer in die Siedlungsgeschichte des Gebiets einzudringen, steht uns eine ungewöhnlich lange Serie von osmanischen Volkszählungs- und Steuerregistern zur Verfügung, die auch das demographisch so unbekannte 18. Jahrhun-

2 Karten: Kanitz, Donau-Bulgarien III, im Anhang. Österreichische Generalkarte OstEuropa 1: 200.000, Wien 1901/03, Blatt 43-44, Svištov. Deutsche Heereskarte 1941, Blatt IV, 5 und 6, Blatt V, 5 und 6. Russische Heereskarte 1879/80. Ein nützliches Werkzeug bei der Identifizierung der einzelnen Dörfer ist Koledarov / Mičev 1973. 
dert abdecken. ${ }^{3}$ Im Folgenden werden wir die Karten, die osmanischen Register sowie historische und archäologische Daten zur Herstellung einer einigermaßen kompletten Totalübersicht verwenden.

Archäologische Ausgrabungen ${ }^{4}$ haben ergeben, dass die Stadt Svištov im 10. Jahrhundert entstanden sein muss und das ganze Mittelalter hindurch existierte. Donau-Bulgarien gehörte von der Mitte des 10. Jahrhunderts bis $1189 \mathrm{zu}$ Byzanz, das sich sehr bemüht hat, seine Nordgrenze die gesamte untere Donau entlang neu zu befestigen und die Gründung von Städten zu fördern. Svištov wird Mitte des 12. Jahrhunderts durch el-Idrisi (st. 1166) zum ersten Mal, unter dem Namen „Subestkastro“, erwähnt, was wohl eine Korrumpierung des griechischen Sebastokastro sein dürfte. In der heutigen bulgarischen Form, als „Svista“, wird die Stadt erst 1385 durch den Reisenden Peter Sparnau erwähnt. Vier Jahre später, im Winter 1389/90, wurde die Burg „Ziştova“ von einer osmanischen Armeegruppe unter Großwesir Candarlı Ali Pascha ohne Kampf in Besitz genommen.

Das prä-osmanische Svištov, von der Städtehistorikerin Margarita Harbova rekonstruiert, bestand aus einem kleinen, auf einem steilen Hügel gelegenen Kastell mit einer offenen Vorstadt an seinem Fuß. ${ }^{6}$ Diese Vorstadt bedeckte ein Areal von kaum mehr als drei Hektar. Wenn wir die bekannte Faustregel von

3 Quellen: OAK 45/29 in der Sofioter Nationalbibliothek für 1479, ein İcmal (synoptisches) Register, veröffentlicht in Todorov / Nedkov 1966, 160-333. MAD 11, im Başbakanlık Osmanlı Arşivi (= BOA), Istanbul, von 1516, ein nicht vollständiges Mufassal (detailliertes) Register.T.D. 370 von 1530 aber basiert auf MAD 11, B.O.A., einem vollständigen İcmal, veröffentlicht als: 370 Numaralı Muhâsebe-i Vilâyet-i Rûm-İli Defteri (937 /1530). Ankara 2002, 505-56.T.D. 416, B.O.A. ein komplettes Mufassal aus der Zeit um 1550.T.D. 718, B.O.A. von1579/80, Kopie eines in Ankara, Tapu ve Kadastro Genel Müdürlüğü liegenden vollständiges Mufassal Registers. Zwei detaillierte Kopfsteuerregister (Cizye) von 1591 und 1601. T.D. 775, (B.O.A.), ein Mufassal'Avariz Register von 1642.T.K.G.M. (Ankara) „Vakf-i Cedid No 144“ von 1710, ein völlig neues Tahrir, erstellt nach den Methoden der klassischen Register aus dem 15.-16. Jahrhundert, enthält die Stadt und 12 ihrer Dörfer. Ein detailliertes 'Avariz Defter von 1751. Die Temettüat Defter 15942-15972 und 12654-12727 aus 1845, und zum Schluss die detaillierten Dorflisten, enthalten im amtlichen Jahrbuch (Salnâme) des Donau-Vilâyets von 1290 (1873) mit Angaben über Haushaltszahlen, Zahl der männlichen Einwohner und der pro Dorf zu zahlenden Steuern.

4 Vor allem viele Münzfunde, byzantinische vom Ende des 10. bis zum 13. Jahrhundert und bulgarische aus dem 13. und 14. Jahrhundert, sowie bulgarische Sgrafitto-Keramik, beides auch aus Oberflächefunden. Für die Ausgrabungen siehe: Vălov 1958, 7-15. Eine reiche Übersicht über die Geschichte der Burg von Svištov geben Kuzev / Gjuzelev 1981, 149-54. Für den Originaltext s.: „Die Jerusalemfahrt des Peter Sparnau und Ulrich von Tennstaedt (1385)“, in: Zeitschrift der Gesellschaft für Erdkunde, 1891 (Berlin), S. 479-491; Hg. Reinhold Röhricht.

5 Zu el-İdrisi siehe: Nedkov 1969, 79. Zu Sparnau s. Kuzev 1970, 68-69.

6 Harbova 1979, 67-68. 
150 Einwohnern pro Hektar anwenden, kommen wir, einschließlich der Garnison, auf etwa 500-600 Einwohner. Wie wir sehen werden, war und blieb Svištov aber bei weiten die größte Siedlung des Gebiets, vier bis fünf mal größer als das größte Dorf seines Bezirks. Bis 1595 entwickelte sich die Stadt stark, wuchs auf 411 von Zivilisten bewohnte Häuser (etwa 2.100 Einwohner) an, wovon nur 70-80 Muslime waren. Am Ende der osmanischen Periode, 1873, hatte die Stadt etwa 13.500 Einwohner, die zu mehr als einem Drittel muslimisch waren.

Während des Kreuzzugs gegen Varna 1444 zog eine riesige Kreuzfahrerarmee durch Donau-Bulgarien und verursachte große Zerstörungen. Ein für das Svištover Land noch verheerenderes Ereignis war der Vernichtungsfeldzug des Vojvoden der Walachei, Vlad des Pfählers, des historischen Prototyps von Graf Dracula. Der Feldzug führte entlang der gesamten unteren Donau und hatte zum Ziel, die Walachei gegen türkische Einfälle zu schützen. Vlad wollte damit Nordbulgarien entvölkern und zerstören, um es als Angriffsbasis für die Walachei ungeeignet zu machen, es handelte sich also um „Vorwärtsverteidigung“.

In einem Brief vom 11. Februar 1462 an König Matthias von Ungarn gab Vlad eine Liste von Menschen, die er getötet und deren abgeschlagene Köpfe man gezählt und notiert hatte. In Novgrad, Batin und Pirgos 384 ,Türken und Bulgaren," in Svištov 410, in Nikopol und Gigen 1.138, in Rahovo 1.460, insgesamt 23.889 Menschen ohne Unterschied der Religion. Dass Vlad nicht zu viel behauptet hat, zeigt das osmanische Register von $1479 .{ }^{7}$

Das älteste erhaltene Register von $1479^{8}$ zeigt, kartographisch verarbeitet, die Folgen der kriegerischen Ereignisse auf sehr deutliche Weise. Wir sehen ein halbzerstörtes und stark entvölkertes Land. In den elf noch existierenden Dörfern lebten insgesamt nur 258 Haushalte (durchschnittlich 23 Häuser pro Dorf). In der Stadt Svištov allein aber gab es schon 215 Häuser. Nur in zwei sehr kleinen, $30 \mathrm{~km}$ tief im Innenland liegenden Dörfer, gab es Muslime. Eines der Dörfer hatte einen gut türkischen Namen: Yayc1 Pınar1 (5 Häuser), das andere hatte sechs muslimische Häuser, aber einen gut bulgarischen Namen: Červena Kruša, später nur Červena genannt. Es kann sich hierbei nur um ein mit Muslimen, wohl Türken, neubevölkertes altes Dorf handeln, an dessen Namen man sich noch erinnern konnte. Beim „Brunnen des Bogenschützen“ war das nicht mehr der Fall. Es gab Wasser und eine gute Siedlungsfläche, aber niemanden, der den Namen noch wusste und weitergeben konnte.

7 Das wichtige Dokument ist mehrmals veröffentlicht. S. Bogdan 1896, 81-82. Vgl. a.: Florescu / McNally 1973.

8 Todorov / Nedkov 1966, 230. 


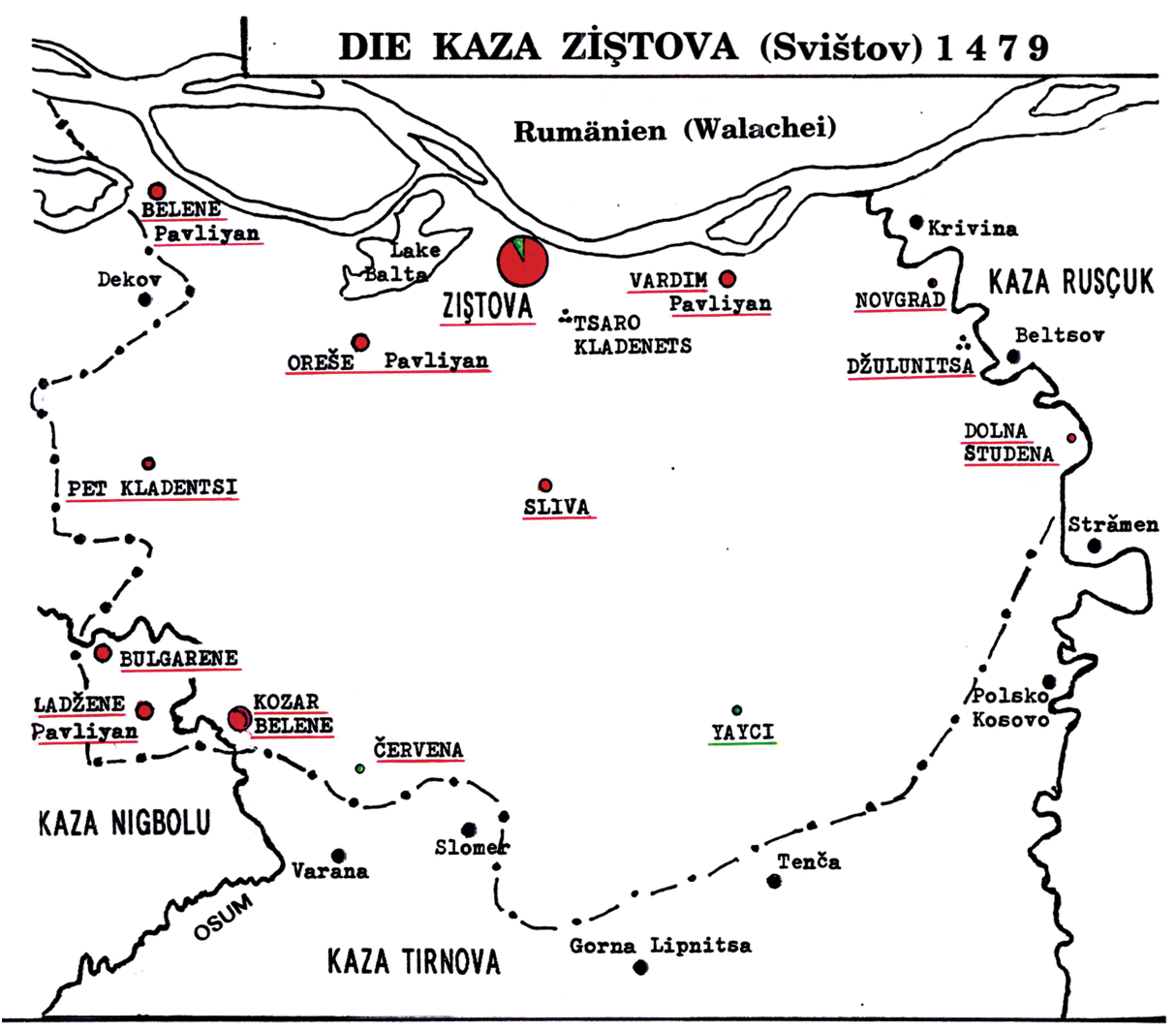

Tenca $=$ Dorf ausserhalb unseres Gebietes

Hier als Orientierungspunkt angegeben
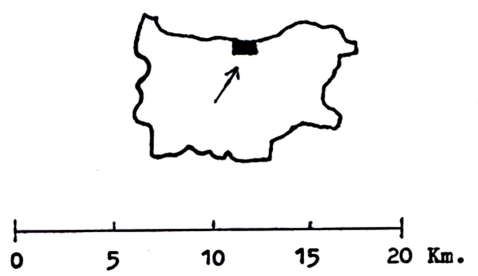

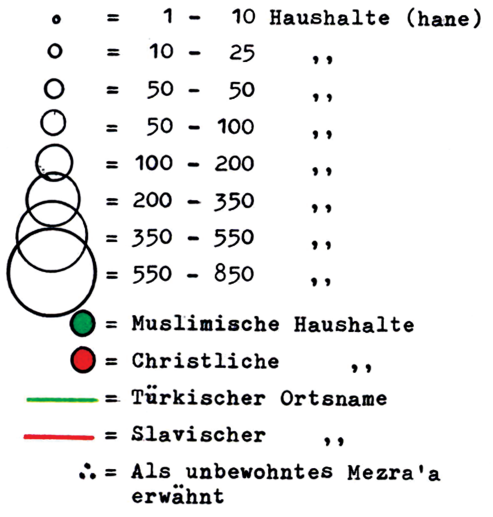

Karte 1, 1479. 
Andere Hinweise auf eine weitgehende Zerstörung in nicht zu fern liegender Zeit bieten zwei Orte mit bulgarischen Namen: Džulunica (Quittenort) am Ufer der Jantra an der Ostgrenze der „Kaza Ziştova“ und Carev Kladenec vier Kilometer südöstlich von Svištov. Beide werden als Mezra'a bezeichnet, also als unbewohnte Orte mit Stücken bebaubaren Landes, das von Bewohnern umliegender Dörfer bearbeitet wurde. Manchmal, aber nicht immer, deutet Mezra'a die Stelle eines zerstörten alten Dorfes an. In den beiden hier genannten Fällen war die Zerstörung nicht all zu lange her, und es gab noch Leute, die den Namen kannten. Im Register ist der zweite Ort mit einer interessanten, erklärenden Notiz versehen:

Die Mezra ‘a Carev Kladenec, nicht im alten Register [erwähnt]. Kâtip Ahmed hat es auf sich genommen, sie neu zu beleben und [im Kriegsfall] einen Soldaten zu stellen. Außerdem wird nichts anderes verlangt, und entsprechend ist es eingeschrieben. Die endgültige Verfügung liegt beim erhabenen Hof [dem Sultan]. ${ }^{9}$

Am Jantraufer lagen noch zwei weitere sehr kleine Ortschaften, Dolna Studena mit acht und Novgrad mit fünf christlichen Haushalten. Die erstere ist entweder die Neubesiedlung einer Mezra'a, oder es handelt sich um überlebende Altsiedler. Novgrad, also Neuburg, wird in der Chronik des Mevlana Neşrî für das Jahr 1388 als Nukesri erwähnt, wohinter sich das griechische Neokastro verbirgt, wohl eine byzantinische Gründung. Vlad der Pfähler hat es auf seiner Liste von Ermordeten. Das Register von 1479 nennt es, wie gesagt, mit fünf Haushalten, wohl Neusiedlern, die sich unterhalb der zerstörten Burg niedergelassen hatten. Auf dem Hügel Kalebayır, außerhalb des heutigen Dorfs Novgrad, liegen die Reste der mittelalterlichen Burg. Auf dem Burggelände fand man byzantinische Münzen aus dem 12. Jahrhundert und Sgrafitto-Keramik aus dem 13. und 14. Jahrhundert, aber keine klaren osmanischen Spuren. Hier unterstützen einander Archäologie und osmanische administrative Quellen gegenseitig.

Es hat ein Register für den gesamten Sandschak Nikopol von ungefähr 1485 gegeben. Leider ist davon nur etwa die Hälfte erhalten geblieben. Es wurde 1997 veröffentlicht. ${ }^{10}$ Die Dörfer von Svištov sind dort nicht enthalten.

9 In der Nähe Novgrads, östlich der Jantra, gibt es ein anderes Beispiel für staatlich durchgeführte Neubesiedlung: das Dorf Marotin. Hier erwähnt Vlads grauenhafte Liste 210 Köpfe, wohl die ganze Dorfbevölkerung.Im Register von 1479 (Todorov / Nedkov II, 198) heißt es: „Das Dorf Marutin, gehörend zu Yerğöğü [= Rusčuk, Ruse]: Weil es mit der Auflage, jeweils 500 akçe Landsteuer [zu bezahlen] und einen Bewaffneten zu stellen, versehen wurde, kam eine Erhöhung. 5 Häuser, Ertrag 657.“ Das Dorf war Teil eines Grosslehens des Yakub Çelebi aus Šumen. Es sieht so aus, als ob kurz nach Vlads verheerendem Feldzug ein neues Register gemacht wurde, das verloren gegangen ist. Yakub Çelebi belebte das Dorf, das 1479 mehr produzierte als 1464/65 und deswegen auch eine höhere Summe zu entrichten hatte.

10 Kovačev 1997. 
Das zweitälteste Register, das das Gebiet von Svištov mit vielen Details beschreibt, ist MAD 11 von 1516. Auf eine Karte transponiert, sehen wir jetzt sehr deutlich, dass es riesige Veränderungen gegeben hat. Statt 11 gibt es jetzt 21 Dörfer. Das gesamte leere Dreieck zwischen Yayc1, Sliva und Dolna Studena hat sich mit neuen Dörfern aufgefüllt. Sechs Dörfer sind mit der Erwähnung versehen, dass sie während der vorherigen Registrierung nicht existierten. Fünf davon haben gute türkische Namen (Akçeyar, Burunlu, Çatma, Habibli und Karaman) und gute muslimische oder alt-türkische Personennamen und Patronyme. Ein neues Dorf hat muslimisch-türkische Bewohner, aber einen bulgarischen Namen, Pavli. Diese sechs Dörfer müssen zwischen 1485 und 1516 entstanden sein. Im Süden der Kaza gab es vier neue Dörfer, die offensichtlich zwischen 1479 und 1485 entstanden sein müssen, weil sie keine erklärende Notiz haben, dass sie nicht in das vorherige Register (von etwa 1485) aufgenommen waren. Es sind Batak, Kara İsa, Koyun Öyük und Sarıyar, also auch gut türkische Ortsund Personennamen. Dazu gab es drei Dörfer mit bulgarischen Namen (Gorna Studena, Stišarov und Vărzulica), ${ }^{11}$ aber mit muslimischen Einwohnern. Diese waren keine zum Islam übergetretenen Bulgaren, wie die Patronymen bezeugen, sondern türkische Neusiedler, die den alten Dorfnamen beibehielten, weil er überliefert war.

Weiter im Norden entstand zwischen 1485 und 1516 noch das Dorf Tatar, oder Tatarköy (Tatarendorf). Diese Tataren sind entweder aus dem Khanat der Krim eingewandert oder kamen aus dem Nordosten von Donau-Bulgarien oder der Dobrudscha, wo es schon seit der vorosmanischen Zeit größere Gruppen tatarischer Einwohner gab. ${ }^{12}$ Zwischen 1479 und 1485 wurde auch Carev Kladenec neu belebt; 1516 hatte es eine gemischte Bevölkerung. Der größere Teil war christlich, der kleinere muslimisch, vier von ihren 13 Haushalten waren ursprünglich Konvertiten.

Neben der Zahl der Dörfer veränderte sich auch die ethnische Zugehörigkeit, ebenso wie die religiösen Verhältnisse. Die Christen in Stadt und Dorf wiesen einen kräftigen Aufschwung, von 380 Häusern 1479 zu 729 im Jahre 1516 auf, sie verdoppelten sich also beinahe. Die Muslime sprangen dagegen von 26 zu 504 Haushalten, verzwanzigfachten sich also! Die Faktor Islamisierung der Lokalbevölkerung, ein so beliebtes Thema der bulgarischen Geschichtsschreibung, kommt kaum in Frage. Von den 504 muslimischen Familienoberhäuptern hatten nur 30 (oder 6\%) keinen muslimischen Vater, waren also Konvertiten der ersten Generation. Die einzige Erklärung ist eine türkische Kolonisationswelle, spontan und nicht staatsgesteuert, da in den Chroniken keinerlei Spuren davon

11 Die Dörfer Stišarov und Vărzulica erscheinen in den osmanischen Quellen in einer, dem Türkischen angepassten Form: İstiçar und Virzil.

$12 \mathrm{Zu}$ denTataren in der Dobrudscha im 13. Jahrhundert siehe: Kiel 2000. 
zu finden sind. Zwischen 1479 und 1516 kamen 300-400 türkische Kolonistenfamilien in die Kaza Ziştova, ließen sich in dem beinahe leeren Land nieder und vermehrten sich stark.

Zwischen 1516 und dem neuen Tahrir, der um 1550 entstand, entwickelte sich das Gebiet weiterhin kräftig, und es entstanden zwei neue muslimisch-türkische Dörfer, Deli Süle und Hacc1 Musa. Insgesamt lebten jetzt 1.721 Familien im Lande, von denen 46\% muslimisch waren. Interessant ist das Auftauchen eines neuen Dorfes, Slive-i Muslim, oder Tursko Slivovo, sechs Kilometer südwestlich des alten Dorfs. Zwischen 1479 und 1516 hat sich Slive/Sliva von 23 auf 61 Haushalte entwickelt, alle christlich. 1550 hat es sich halbiert, aber ein neues Dorf mit 13 muslimischen Haushalten hat sich im gleichen Zeitraum gebildet. Die Erklärung kann nur sein, dass sich kurz nach 1516 eine Gruppe der bulgarischen Einwohner zum Islam bekehrt und vom Mutterdorf abgespalten hat. Für das frühe Datum der Abspaltung spricht die Tatsache, dass von den 13 Haushalten nur zwei Konvertiten waren. Obschon in anderen Dörfern Muslime und Christen durcheinander und miteinander lebten, hat es im „Pflaumendorf“ offensichtlich Spannungen gegeben. Es erscheint wie Ironie, dass „Tursko Slivovo " 1751 schon zu einem Drittel von Christen besiedelt war, und 1873 zu nicht weniger als 95\%, weil Bulgarsko Slivovo bis Ende der osmanischen Zeit rein bulgarisch geblieben war. Von 366 Haushalten waren nur noch 16 muslimisch. Der Dorfname aber blieb gleich, weil niemand sich damals um solche Dinge kümmerte. Dies steht in schroffem Gegensatz zu den Verhältnissen des von virulentem Nationalismus verseuchten 20. Jahrhunderts.

In den etwa 35 Jahren bis zum Tahrir von 1579/80 wuchs die Gesamtbevölkerung nochmals kräftig und erhöhte sich von 1.721 auf 2.532 Familien. Die Muslime waren deutlich schneller gewachsen als die Christen. Hierbei spielte Konversion zum Islam eine größere Rolle als in den vorhergehenden Jahrzehnten. Jetzt war jeder fünfte muslimische Familienvater ein neubekehrter Muslim. Neben der Konversion spielte jedoch die Familiengröße eine durchschlagende Rolle. Die Muslime hatten erheblich mehr Kinder.

In der zweiten Hälfte des 16. Jahrhunderts was das Gebiet von Svištov als Folge eines mehr als ein Jahrhundert andauernden spontanen Prozesses zu einem mehrheitlich muslimischen Land geworden. 


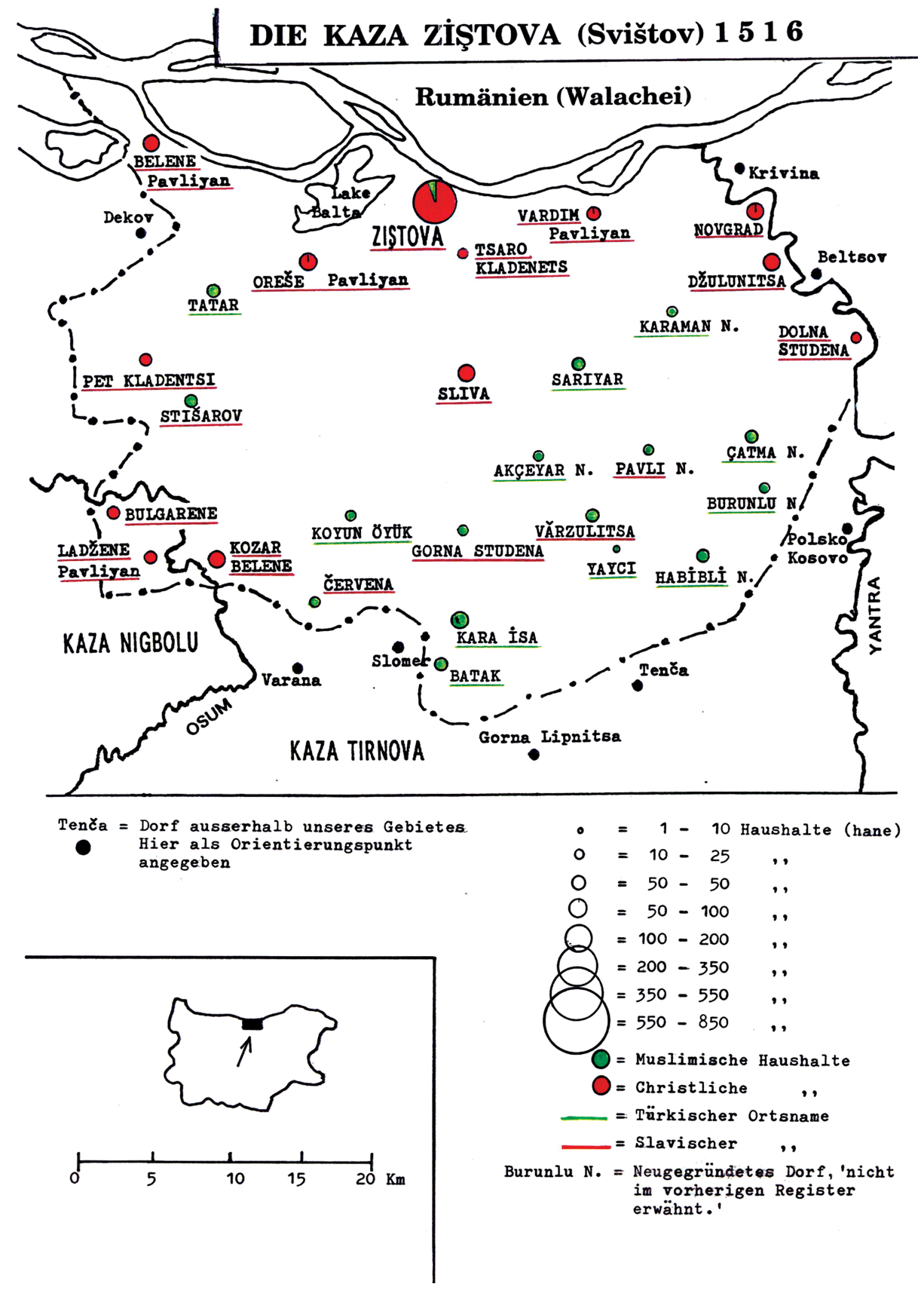

Karte 2, 1516. 


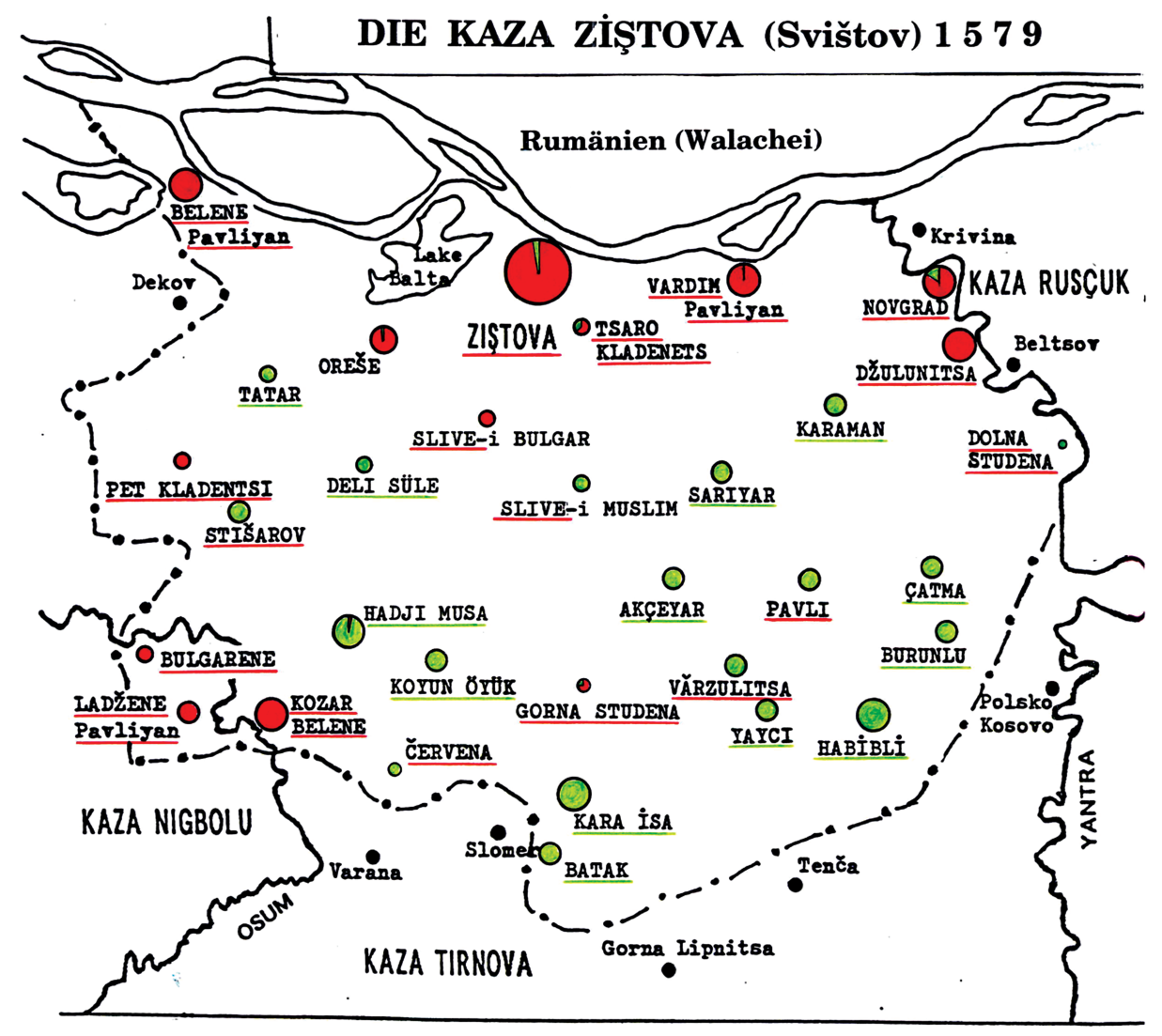

Tenča = Dorf ausserhalb unseres Gebietes. Hier als Orientierungspunkt

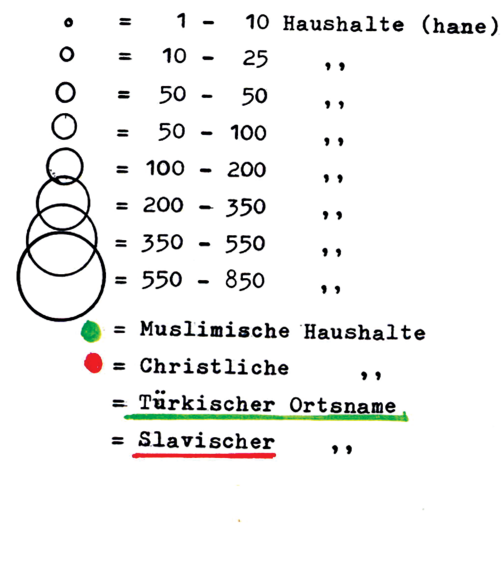

Karte 3, 1579/80. 


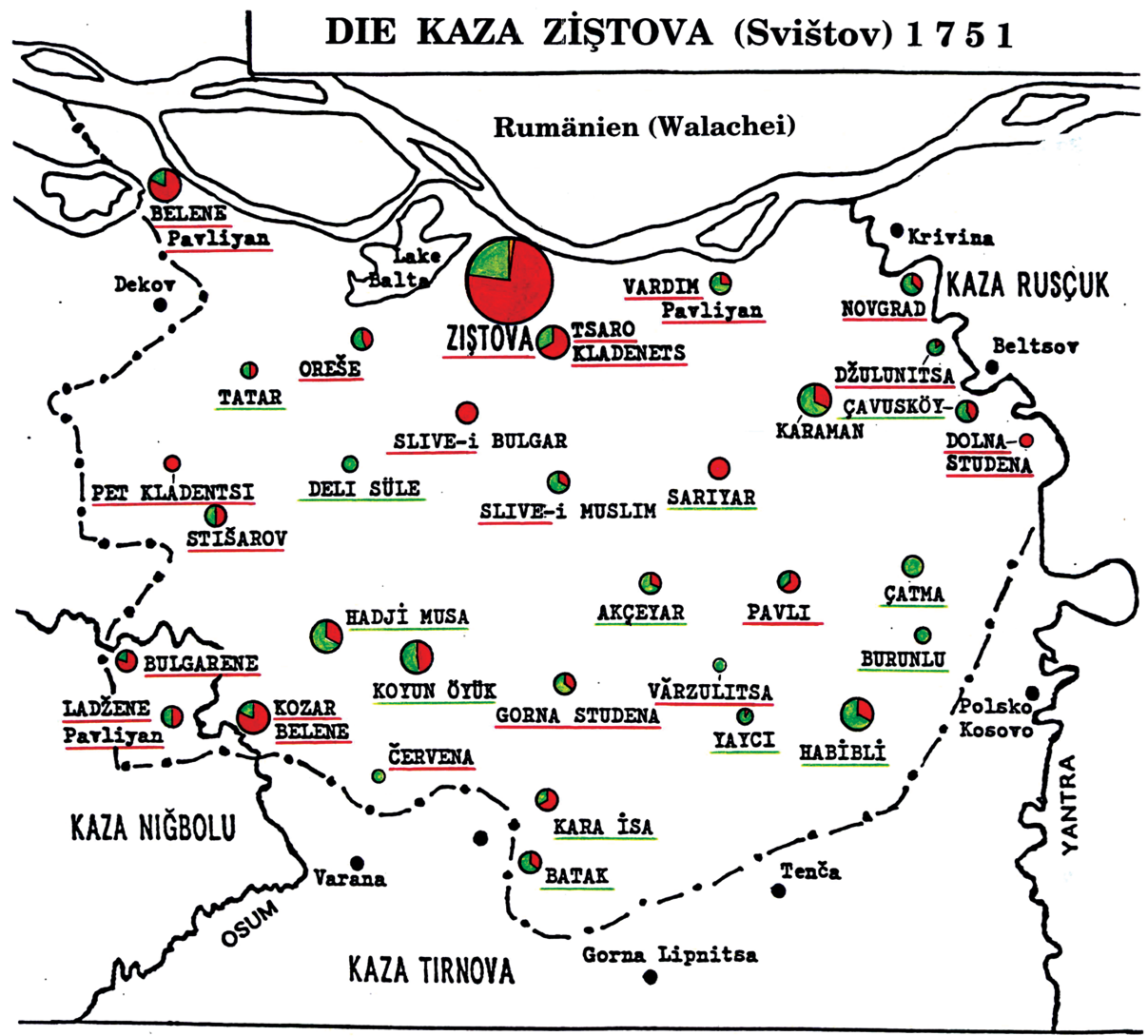

Tenča = Dorf ausserhalb unseres Gebietes

Hier als Orientierungspunt angegeben

$$
\begin{aligned}
& 0=1-10 \text { Eouseholds (Hane) } \\
& 0=10-25 \quad, 1 \\
& 0=25-50 \quad \text { " }
\end{aligned}
$$

( $)$ Jüdische Haushalte

$\mathrm{O}=50-100 \quad$,

$Q=100-200 \quad \cdots$

$Z=200-350 \quad \cdots$
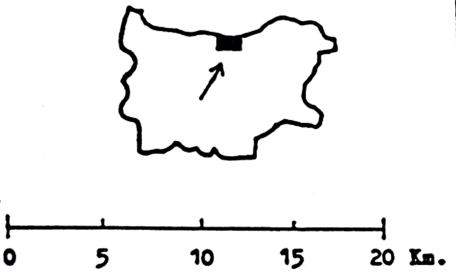

$=350-550 \quad \cdots$

$=550-850 \quad \cdots$

= Muslimische Haushalte

$\mathrm{O}=$ Christliche Haushalte YAYCI = Türkischer Ortsname

Novgrad = Bulgarischer , 
Die genannte Tendenz hätte sich im 17. Jahrhundert noch fortgesetzt, aber es kam zu einem furchtbaren Bruch. Zwischen den genannten Daten ereignete sich zweimal hintereinander eine entsetzliche militärische Katastrophe. Im Jahre 1595 erschien der Fürst der Walachei, Michael der Tapfere, mit einer großen Armee in Nordbulgarien. Er brannte Svištov nieder, konnte aber das Kastell nicht bezwingen. Muslime, deren man habhaft werden konnte, wurden getötet, Christen in die Walachei zwangsumgesiedelt. Drei Jahre später wiederholte sich die gleiche Geschichte. ${ }^{13}$ Erzählende Quellen, die schildern, was auf dem offenen Lande geschah, liegen nicht vor, aber die osmanischen Register legen ein deutliches Zeugnis dieser militärischen Ereignisse ab.

Nach dem Kopfsteuerregister von 1591 wohnten 1.371 christliche Haushalte im Gebiet. Nach dem Register von 1601 waren es nur noch 855. Für 1591 und 1598 liegen über die Muslime keine Zahlen vor, aber im Register der „außerordentlichen Steuern“ ('Avâriz-i Divâniye) vom Jahre 1642 erscheinen sie wieder. Diesmal von 1.367 Haushalten 1580 gesunken auf 1.099 im Jahre 1642, wobei man annehmen darf, dass zwischen 1598 und 1642 sich die Muslime zahlenmäßig kräftig erholt haben.

Bei einem Vergleich zwischen der Situation zwischen 1579 und 1642 erhält man ein sehr unausgewogenes Bild der Veränderungen. Die Dörfer Akçeyar, Habibli und Koyun Öyük hatten sich gut gehalten. Tief gesunken aber waren Kara İsa, Pavli und Saryyar (letzteres von 87 Haushalten, alle muslimisch, auf 20, ebenfalls alle muslimisch). Demgegenüber stieg Karaman von 75 auf 92 Haushalte, davon einer christlich. Der Niedergang der türkischen Kolonistendörfer wird am extremsten durch Kara İsa illustriert. 1579 hatte es 140 Haushalte, alle Muslime, davon nur sieben Muslime der ersten Generation, und kein einziger Christ. 1642 hatte sich das Dorf mit 77 Haushalten halbiert, 27\% davon waren jedoch christlich. Diese Entwicklung setzte sich stark durch. Das detaillierte 'Avâriz-Register von 1751 zeigt, dass die Verhältnisse sich jetzt umgedreht hatten: statt $27 \%$ gab es jetzt $67 \%$ Christen! Das Dorf entwickelte sich weiter und erreichte 1845 wieder den Stand von 1579. Es war jetzt aber zu 93\% christlich.

Ein etwas anderes Bild zeigt sich in den alten Paulicianer-(Bogomilen-) Dörfern. In „Belene-i Pavliyan“ nennen die Register von 1479 bis 1579 keinen einzigen Muslim in diesem stets großen Dorf. 1642 gab es neun muslimische Haushalte, 1710 dann 34. Daraufhin stoppte der Prozess der Islamisation. 1873 zählte Belene 285 Haushalte und war so groß wie nie zuvor. Die Muslime erreichten im letztgenannten Jahr kaum 10\%. Zwischen 1579 und 1642 sank die

13 Die Dokumentation zu diesen Ereignissen ist gesammelt und veröffentlicht durch Hurmuzaki 1906, 25, 415. Im Vergleich mit den osmanischen Quellen lässt sich leicht feststellen, dass die Zahlen der in die Walachei verschleppten Bulgaren maßlos übertriebene Kriegspropaganda ist. 
Bevölkerung von Oreše-i Pavliyan kaum, aber im letztgenannten Jahr war die Bevölkerung zu 9\% muslimisch und 1751 zu 59\%. Lădžene-i Pavliyan hatte 1579 noch keine Muslime, 63 Jahre später war es allerdings zu 31\% islamisch und $1710 \mathrm{zu}$ 46\%. Im 19. Jahrhundert drehte sich der gesamte Prozess um, und 1873 war das Dorf nur zu 17\% islamisch.

Im mittleren Donau-Bulgarien gab es seit dem vor-islamischen Mittelalter Dutzende Bogomilensiedlungen, erkennbar am Epitheton „Pavliyan“ (PaulusChristen). Diese Gruppe hatte durch die bulgarische orthodoxe Kirche schwere Verfolgungen zu erdulden. Aus den Berichten der päpstlichen Missionare zwischen 1565 und 1799, 1887 von Eusebius Fermendžin veröffentlicht, ist ersichtlich, dass es den Türken völlig gleichgültig war, was diese Paulicianer glaubten. Nach den Worten Jirečeks genossen die „Pavlikeni“ völlige Religionsfreiheit. Vor allem im 17. Jahrhundert betrieb die katholische Kirche eine rege Missionstätigkeit unter den Paulicianern. Aus den Visitationsberichten wissen wir, dass sich die Paulicianer im Laufe des 17. Jahrhunderts zur römisch-katholischen Kirche bekehrten. Von den Missionaren unerwähnt, aber aus den osmanische Registern klar ersichtlich, nahm im Laufe der Zeit ein Viertel oder ein Fünftel der Paulicianer den Islam an. In dreien der vier Pavlikenidörfer in der Kaza Svištov (Belene, Lădžene und Oreše) gab es 1579 keinen einzigen Muslim. Im Jahre 1642 waren die Ortschaften aber zu einem Zehntel muslimisch und 1873 zu 18\%. Anders gestaltete sich die Entwicklung des Dorfes Vardim am Donauufer. Schon 1579 gab es dort drei muslimische Haushalte, alle Konvertiten, 1642 aber 44. Bis 1873 nahm die Zahl der katholischen Christen langsam zu, die Muslime bildeten jedoch noch immer 57\% der Gesamtbevölkerung des Dorfes. Die Tatsache, dass ein erhebliches Teil der paulicianische Bevölkerung Donau-Bulgariens nicht den Islam annahm, sondern das römisch-katholische Christentum, setzt ein großes Fragezeigen hinter die landläufige Theorie, dass die Balkanchristen im 17. Jahrhundert den Islam annahmen, um der Kopfsteuer zu entgehen und ihren gesellschaftlichen Status zu verbessern. Die bulgarischen Paulicianer taten genau das Gegenteil. Dazu kam der glühende Hass des orthodoxen Klerus, der wo immer möglich, die Katholiken bei den osmanischen Behörden als Spione des Papstes verleumdete. ${ }^{14}$

Im Jahre 1710 wurde in der Istanbuler Vorstadt Üsküdar eine große neue Moschee und ein 'Imaret (Speisehaus für Arme und Reisende) fertig gestellt. Bauherrin war Gülnuş Ümmetullah, Mutter des Sultans Ahmed III. Für den Unterhalt dieser Gebäude wurde eine Stiftung gegründet, die als Besitz die Steuereinkünfte der Stadt Svištov sowie von 12 seiner Dörfer geschenkt erhielt. Diese Statusänderung war von Bedeutung für Stadt und Dörfer, da sie im all-

14 Jireček 1891, 108-111; Fermendžiu 1887, 9-87. 
gemeinen mit Privilegien verbunden war. Die Oberaufsicht über die großen Stiftungen hatte der Kızlar Ağa, der Obereunuch, der bibliophile Haccı Beşir A ğa. Das ist wohl die Erklärung dafür, dass gerade diese Person in Svištov eine Bibliothek mit arabischen, türkischen und persischen Handschriften gründete. Der Stiftungsverwalter (vom Kızlar Ağa ernannt) und Vojvode der Stadt, Hüseyin A $\breve{a}$, fügte 1765/66 einen monumentalen Uhrturm hinzu. Die Statusveränderung Svištovs und seiner Dörfer hatte zur Folge, dass 1710 eine neues, sehr detailliertes tahrir gemacht wurde (Ankara, T.K.G.M. Vakf-i Cedid 144). Zusammen mit dem detaillierten mufassal (Register der Avariz-Steuer) von 1751 erlaubt es dem Historiker, auch in das, demographisch gesprochen, so wenig bekannten 18. Jahrhundert vorzudringen.

Die allgemeinen Linien, sichtbar in den Zahlen für die erste Hälfte des 18. Jahrhunderts, also die relative Abnahme der Zahl der muslimischen Dorfbewohner und die Umschichtung der Stadtbevölkerung, wurde anschließend durch die vier entsetzlichen Russisch-Türkischen Kriege 1768-74, 1788-92, 180610 und 1827-28 noch verstärkt. Die meisten türkischen Kolonistendörfer, die im 16. Jahrhundert ausschließlich türkisch gewesen waren und nur sehr wenige Konvertiten beherbergten, verloren ihren muslimischen Charakter. Bulgarische Christen ließen sich in diesen Dörfern nieder und wurden akzeptiert, obwohl sie sich nicht zum Islam bekehrten. 1844/45, wo wir wieder eine komplette Zahlengruppe haben, waren viele türkische Kolonistendörfer mehrheitlich christlich-bulgarisch besiedelt.

Eine der wesentlichen Ursachen, warum die Zahl der muslimischen Dorfbewohner sank, muss im Umzug in die Stadt gesucht werden, wo man größere Sicherheit erwartete. Vor dem Rückzug am Ende des russisch-türkischen Kriegs von 1806-1810 befahl Oberbefehlshaber Michail Kutuzov, Svištov und die anderen Donaustädte in russischen Händen zu vernichten. Die Festung wurde gesprengt und die Stadt (damals angeblich mit etwa 15.000 Einwohnern) brannte nieder. ${ }^{15}$ Als Handels- und Handwerkstadt erholte sich Svištov sehr schnell, aber es veränderte sich durch Zuzug aus den Dörfern und aus der Stadt Tărnovo und ihrem Satelliten Arbanasi stark. Auch jüdische Händler ließen sich in der Stadt nieder. Um 1845 war der Prozentsatz der Muslime in den Dörfern auf 35\% gesunken, in der Stadt hatte er indes sehr stark zugenommen. 1751 hatte Svištov 19\% Muslime gehabt, 1845 jedoch 44\% und 1873 48\%. Hier werden die Folgen zweier vernichtender Kriege auf dramatische Weise sichtbar.

15 Zinkeisen 1863, 702-706. 


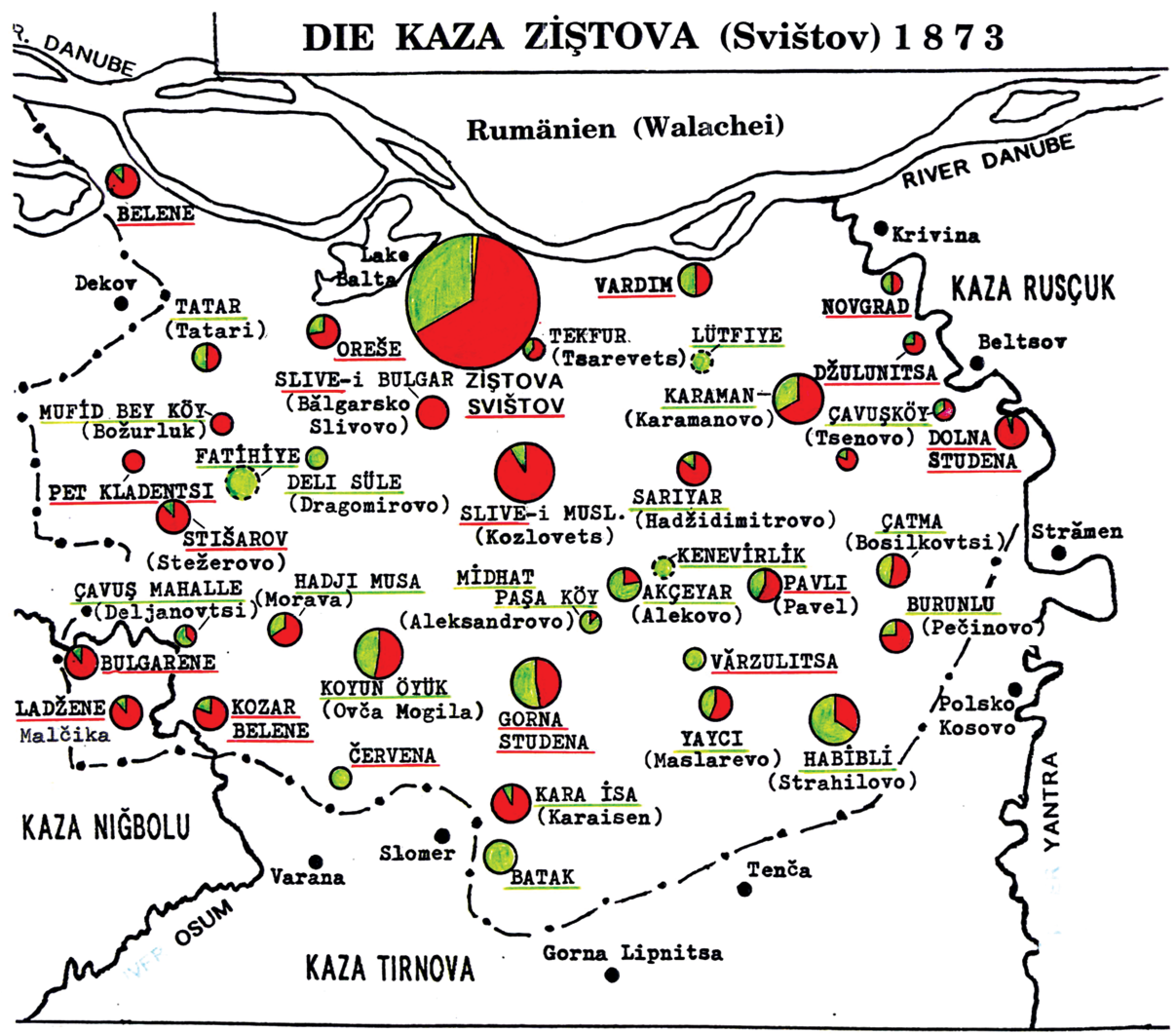

$$
\begin{aligned}
& \text { Biberli/Piperkovo }=\begin{array}{c}
\text { parallel benutzte } \\
\text { Namensform }
\end{array} \\
&(--)= \begin{array}{l}
\text { Moderne Umbenennungen } \\
\text { (fast alle 1934) }
\end{array} \\
&= \text { Nach } 1878 \text { verschwundenes Dorf. } \\
& \text { Lage nur ungefähr bekannt } \\
& \text { Tença = } \text { Dorf ausserhalb unseres Gebietes } \\
& \text { Hier als Orientierungspunkt } \\
& \text { angegeben } \\
& \text { W }= \text { Jüdische Haushalte }
\end{aligned}
$$
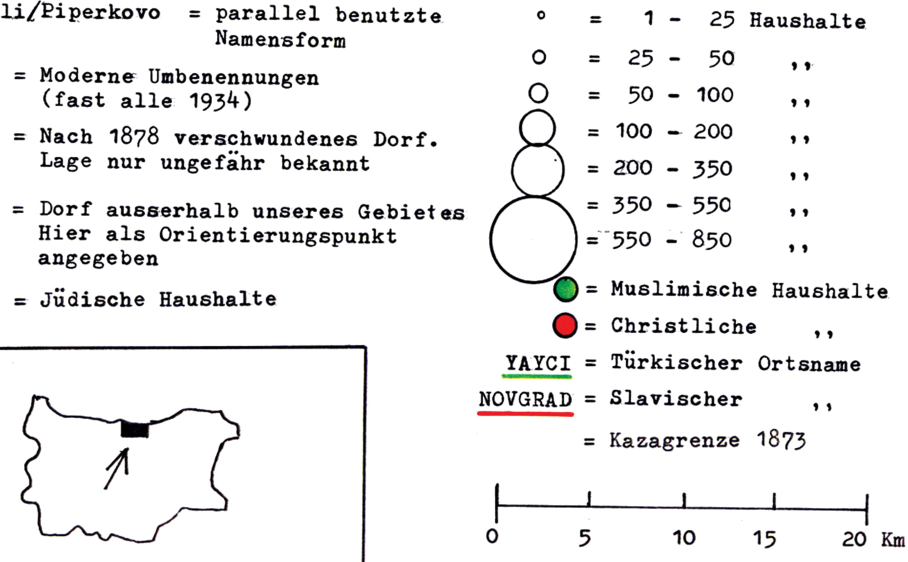

Karte 5, 1873. 
Nach dem Krimkrieg (1852/54) bemühte sich die osmanische Regierung sehr, die ethnisch-religiöse Lage im offenen Land Donau-Bulgariens zu Gunsten des staatstragenden Volkes zu verändern. Vier neue Dörfer wurde gegründet: Fethiye und Kenevirlik mit 206 abchasischen Flüchtlingsfamilien aus dem Kaukasus, sowie Midhat Paşa und Lütfiye mit 149 tscherkessischen Haushalten, ebenfalls aus dem Kaukasus. Daneben wurde das alte Muslimdorf mit dem bulgarischen Namen Červena mit 72 tscherkessischen Haushalten neubesiedelt. In der bulgarischen Historiographie ist es geläufig, die Ansiedlung muslimischer Flüchtlinge als gefährliche Bedrohung für das Bulgarentum dazustellen. 1845 waren 35\% der Dorfbewohner Muslime, 1873 43\%. Ziehen wir für das Jahr 1873 die Flüchtlinge ab, bleiben 38\%. Die muslimische Landbevölkerung hat also durch die Ansiedlung lediglich um 5\% zugenommen.

Am Vorabend des verheerenden russisch-türkischen Kriegs von 1877/78 beschreiben die spätosmanischen Salnâmes sowie einige europäische Forscher Svištov als eine Stadt mit ungefähr 12.000-13.000 Einwohnern, 19 Moscheen, 4 Kirchen, einer Synagoge, einem Bad (hamam), 18 Karavansarays, 738 Geschäften und 170 Speichern. Die Kaza umfasste 40 Dörfer und hatte zusammen mit der Stadt 3.580 muslimische und 4.450 nicht-muslimische Häuser. Die Stadt selbst war zu etwas mehr als einem Drittel seiner Bevölkerung muslimisch. ${ }^{16}$

Nach der bulgarischen Unabhängigkeit 1878 änderte sich das Bevölkerungsbild von Svištov und seinem Gebiet drastisch. Der Großteil der Muslime verließ die Dörfer und übersiedelte nach Anatolien. Innerhalb der Stadt verschwand in wenigen Jahren die Hälfte der Muslime. Die Entwicklung der Stadt stagnierte. 1934 betrug die Bevölkerungszahl 12.082, also weniger als im Jahr 1887. ${ }^{17}$ Erst nach dem Zweiten Weltkrieg wuchs die Stadt wieder, 1972 hatte sie 23.182 Einwohner, heute rund 70.000. Davon war nur eine kleine Minderheit muslimisch und turkophon. Von den 19 Moscheen die Svištov bis 1877 gehabt hatte, stehen noch zwei. Von der im frühen 18. Jahrhundert gestifteten Bibliothek des Haccı Beşir Ağa und allen ihren Büchern fehlt jede Spur. Die sechs Kirchen sind indes noch alle da. Auf dem Land gibt es außerhalb des Großdorfs Batak fast keine Muslime mehr, und Moscheen, Derwisch-konvente oder islamische Schulgebäude sind verschwunden. Ein halbes Jahrtausend Siedlungsgeschichte unseres Bezirks ist abgeschlossen, rückgängig gemacht und fast vergessen.

16 Für Auszüge aus dem amtlichen Jahrbüchern in deutscher Übersetzung siehe die höchst informative Arbeit von Kornrumpf 1976, 363. Die Zahl der Kirchen in Svištov ist nicht genau. Čankov (siehe unten) gibt die Namen von sechs Kirchen in Svištov, alle mit Baudatum: Sv. Dimităr die älteste, Peter und Paul von 1644, Prorok Ilija, erneuert 1835, Preobraženie 1835-36, Sv. Troica 1865-67, und schließlich Kiril und Metodi von 1874, ein schöner Spiegel der osmanischen Religionspolitik.

17 Für die Bevölkerungszahlen und Kirchen siehe: Čankov 1939, 391-92. 
Das hier Gebotene ist lediglich ein Baustein im reichen Mosaik der osmanischen Siedlungsgeschichte und Urbanisierungspolitik. Dieses bunte Mosaik einigermaßen zu vervollständigen, sollte eine wichtige Forschungsaufgabe für uns sein, denn: ohne Bevölkerung gibt es keine Geschichte. Bevor eine derartige Aufgabe jedoch realisiert ist, gibt es noch sehr viel zu tun.

\section{Literaturverzeichnis}

370 Numaralı Muhâsebe-i Vilâyet-i Rûm-İli Defteri (937 /1530). Ankara 2002.

Bogdan, Ioan, 1896: Vlad Tepeş şi narațiunile Germane şi Rusesți asupra lui. București.

Čankov, Žečo, 1939: Geografski Rečnik na Bălgarija. Sofia.

Fermendžiu, Eusebius, 1887: Acta Bulgariae Ecclesiastica. (Monumenta spectantia Historiam Slavorum Meridionalum, Vol. 28). Zagreb.

Florescu, Radu / McNally, Raymond, 1973: Dracula: A biography of Vlad the Impaler. New York (Hawthorn Books).

Harbova, Margarita, 1979: Ukrepenijat Bălgarski srednovekoven grad, XIII-XIV vek. Sofia.

Hurmuzaki, Eudoxiu de, 1906: Documente privitoare la istoria Romanilor, XII. Bucureşti.

Jireček, Constantin,1891: Das Fürstenthum Bulgarien. Wien.

Kiel, Machiel, 2000: „Sar1 Saltuk: Pionier des Islam auf dem Balkan in 13. Jahrhundert,“ in: Ergin, Ismail / Franz, Erhard (Hrsg.): Aleviler/Alewiten, Identität und Geschichte. 253-286.

Koledarov, Petr / Mičev, Nikolaj, 1973: Promenite v Imenata i Statuta na Selištata v Bălgarija, 1878-1972. Sofia.

Kornrumpf, Hans Jürgen, 1976: Die Territorialverwaltung im östlichen Teil der europäischen Türkei, 1864-1878. Freiburg.

Kovačev, Rumen, 1997: Opis na Nikopolski Sandžak ot 80te godini na XV vek. Sofia.

Kuzev, Aleksandar, 1970: „Pătepisni beležki za bălgarskite zemi ot 1385 g.“, in: Arheologija XII, 4, 1970, 68-69.

Kuzev, Aleksandar / Gjuzelev, Vasil, 1981: Bălgarski srednovekovni gradove i kreposti, Tom I: Gradove i kreposti po Dunav i Crno More. Varna.

Nedkov, Boris, 1969: Bălgarija i săsedinite i strani prez XII vek spored „Geografijata“ na Idrisi. Sofia.

Todorov, Nikolai / Nedkov, Boris (Hrsg.), 1966: Turski Izvori za Bălgarskata Istorija, II. Sofia.

Vălov, Vălo, 1958: „Razkopki na Kaleto v grad Svištov“, in: Arheologija IV, 4, 1958, Sofia, 7-15.

Zinkeisen, Johann W., 1863: Geschichte des osmanischen Reiches in Europa,VII, Gotha. 
